\title{
Eradication of HIV-1 from CNS reservoirs: current strategies and future priorities
}

\author{
Jeymohan Joseph
}

Received: 23 February 2015 / Accepted: 25 February 2015 / Published online: 5 May 2015

(C) Journal of NeuroVirology, Inc. (outside the USA) 2015

Eradication of HIV-1 and achieving a sterilizing or functional cure has become a priority area in the AIDS field. Large investments have been made by funding agencies, particularly $\mathrm{NIH}$, in understanding the mechanisms of HIV persistence and approaches to target and eradicate latent reservoirs. The majority of the work that is ongoing has focused on studying resting CD4+ $\mathrm{T}$ cell reservoirs and developing strategies for reactivation and purging HIV from this cell type. However, it is becoming increasingly clear that other sites of HIV persistence exist including anatomic reservoirs such as the brain and gut. Also, myeloid cells residing in these anatomic compartments may harbor persistent HIV-1 that could potentially be a source of rebounding virus upon cessation of therapy.

The brain is unique in terms of its immune privileged status and the presence of the blood-brain barrier which restricts entry of anti-retrovirals. HIV-1 seeds the brain early in infection and may reside in long-lived cells such as microglia and astrocytes and thus serve as a site of viral persistence. At an NIMH-sponsored meeting entitled "Eradication of HIV-CNS Reservoirs-Current and Future Strategies" held in Washington DC in conjunction with the 12th International Symposium on NeuroVirology (October 2013), many of the challenges in targeting brain HIV-1 reservoirs and the need to better understand the mechanisms of establishment of latency in this unique anatomic compartment were discussed. At that meeting, it was felt that the field would greatly benefit by the publication of a special issue of the Journal of NeuroVirology

J. Joseph $(\bowtie)$

Division of AIDS Research, National Institute of Mental Health, Room 9G20, MSC 98315601 Fishers Lane,

Bethesda, MD 20892-9830, USA

e-mail: jjeymoha@mail.nih.gov

J. Joseph

Rockville, MD 20852, USA

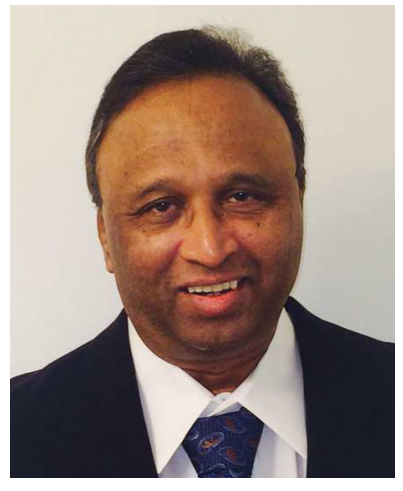

devoted to HIV-1 CNS reservoirs that would address the challenges and current knowledge in this area. This special issue represents work presented by many of the speakers at the NIMH-sponsored symposium. In addition, several other authors have contributed in order to provide a comprehensive review of the state of the field. The articles presented discuss current challenges in targeting CNS reservoirs as well as mechanisms of establishing persistence in CNS-derived cell populations such as microglia, macrophages, and astrocytes. Current approaches to target myeloid reservoirs as well as some novel gene editing strategies that may aid in HIV-1 eradication strategies are also discussed. I have provided below, for the reader of this special issue, a brief summary of each of the papers published in order to capture the flavor of the topic area covered. The summaries are by no means comprehensive but are illustrative of the research areas that these papers represent.

An overview of the translational challenges in targeting latent HIV infection and the CNS reservoir problem was highlighted by Dr. David Margolis. Some of the challenges include the need for reliable, validated cell-based and animal model systems to test latency-reversing agents. He cautions that of several potential anatomic reservoirs, the central 
nervous system represents a significant obstacle since it is difficult to access for study and therapeutic intervention. Dr. Margolis also outlined some of the current clinical trials using latency reactivation agents such as vorinostat. Generally, outcomes for these trials are evaluated using peripheral blood samples since the blood is the most accessible compartment and represents systemic effects. However, he stressed that it is important to evaluate treatment outcomes in other compartments such as the CNS to ensure that neurocognitive outcomes are not impaired as an unwanted side effect of viral induction strategies.

Dr. Avindra Nath provided an overview of the different cell populations that serve as potential reservoirs of HIV in the brain, issues related to eradication of the virus from sanctuaries in the brain, and challenges faced by neuroscientists in finding a cure. He expressed concerns about current reactivation strategies being tested. Reactivation of virus in the brain could lead to infiltration of cytotoxic T cells leading to devastating encephalitis termed CNS-immune reconstitution syndrome, which could be injurious to the neurons. He also cautioned that whatever strategy is used to achieve a sterilizing or functional cure, it is critical that close attention be given to controlling the CNS viral reservoir or else there would be a theoretical risk for reseeding the periphery with virus from the CNS.

Here, we also incorporated an article by Drs. Jay Rappaport and David Volsky where they discuss the role of macrophages in HIV-associated neurocognitive disorders as well as a reservoir for latent HIV. They emphasize the importance of monocyte/macrophage homeostasis, immune polarization and the interferon response in neurocognitive impairment, and immune dysfunction remaining in successfully treated patients. The authors underscore the urgent need for pharmacologic strategies to effectively modulate inflammation and immune polarization.

The strategy toward the role of macrophages in virus-host interplay and features of this interplay that could impact efforts to eliminate myeloid cell reservoirs were presented by Dr. Mario Stevenson. He indicated that despite numerous demonstrations that macrophages can be infected with primate lentiviruses in vitro, there has been little attention or interest in establishing whether macrophage reservoirs are important for the maintenance of infection, particularly in the face of antiretroviral suppression. Also, Dr. Stevenson described some of the literature related to myeloid cell restriction factors such as SAMHD1 and strategies used to overcome this restriction, such as vpx. This review also discusses obstacles to the elimination of myeloid cell reservoirs. It is not clear that myeloid cell reservoirs would be amenable to elimination by the approaches that are being explored to eliminate CD4+ T cell reservoirs, and prior research suggests that they might not. Monocyte-derived macrophages (MDM) have been shown to be resistant to HIV-1 cytopathicity. Moreover, strategies to induce cytopathicity by potential use of antagonists (Gleevec) to survival cytokines such as monocyte colony stimulating factor (MCSF) were highlighted in this presentation. Finally, this review discusses the potential use of alveolar macrophages to provide an important window into the dynamics of macrophage reservoirs under different treatment regimens and whether viruses in tissue macrophages from individuals on suppressive ART contribute to viral persistence.

Dr. Tracy Fischer provided evidence that macrophages comprising perivascular cuffs and nodular lesions in SIV encephalitis (SIVE), which are likely the primary reservoir for productive SIV in the brain, also serve as the principal source for M-CSF. She also demonstrated that M-CSF and IL-34 which signal through the same receptor, cFMS, enhance HIV-1 production by microglia in vitro. This can be targeted by the addition of a receptor tyrosine kinase inhibitor with high specificity for cFMS, GW2580. Based on this data, Dr. Smith suggested that cFMS signaling may provide an attractive target for eliminating long-lived macrophage reservoirs for HIV in the brain and other tissues.

Results from Dr. Fatah Kashanchi's laboratory provide new evidence that a Tec family tyrosine kinase is upregulated in HIV-1-infected cells. The group has previously identified the Bruton's tyrosine kinase (BTK) as one of 17 candidate proteins unique to latent HIV-1-infected T cells. Here, they expand their findings to myeloid cells and show that BTK can be inhibited to decrease virus production and increase cell death. BTK was originally identified in B cells and it has been found more recently in myeloid cells, including monocytes, macrophages, neutrophils, and mast cells. Unique in this work is the finding that BTK is phosphorylated, and using size exclusion chromatography, they found that BTK protein complexes were distributed in both a high molecular weight $(\sim 600 \mathrm{kDa})$ and a low molecular weight complex $(\sim 60$ $120 \mathrm{kDa})$ in infected U1 cells. Finally, the group utilized a number of biochemical and high-throughput Western blot analyses (RPMA) to show that BTK-specific antibody and small molecule inhibitors, including LFM-A13 and FDAapproved ibrutinib (PCI-32765), can sensitize infected cells to apoptosis and decrease virus production.

Potential HIV-1 target cells in the CNS with an emphasis on the evolution and tropism of viruses that infect macrophages and microglia in the brain were discussed by Dr. Sarah Joseph. Severe neurocognitive impairment is associated with sustained replication in macrophages in the CNS. It is believed that late in the disease, in the CNS of some individuals, HIV lineages adapt to infecting macrophages - a process that produces viruses that are able to efficiently enter macrophages and other cells expressing low levels of CD4. Dr. Joseph stated that despite the fact that most viruses are welladapted to infecting $\mathrm{T}$ cells and poorly adapted to infecting macrophages (i.e., are T cell-tropic), they are still able to infect macrophages at a low level which could potentially contribute 
to the reservoir and set the stage for the evolution of macrophage tropism.

Dr. Melissa Churchill reviewed the unique characteristics of the CNS that may potentially influence the establishment and maintenance of CNS HIV-1 viral reservoirs. She alluded to potential differences between CNS reservoirs and $\mathrm{T}$ cell reservoirs. For instance, CNS-derived viral strains have long terminal repeat (LTR) mutations that are distinct to those found in other anatomical compartments. Secondly, the CNS-specific cell types that are infected with HIV-1 have markedly different expression of cellular transcription factors that could impact the transcriptional activity of the infecting virus. Also, reduced immune surveillance in the CNS will influence treatment outcomes when testing transcriptional activators to purge viral reservoirs. In addition, the altered antiretroviral drug availability in the CNS may potentially hinder the blocking of new rounds of infection. Thus, current viral reactivation strategies being tested may worsen outcomes in the CNS, leading to an expansion of infected cells rather than achieving a reduction in reservoir size.

The current literature on humanized mouse models and their utility in understanding how HIV-1 infection leads to changes in the central nervous system was reviewed by Dr. Victor Garcia-Martinez. He also identifies the opportunities and limitations of existing model systems in the study of mechanisms of establishment of HIV-1 CNS reservoirs in vivo. This paper also touches upon the development of newer humanized mouse models that contain a full complement of T cells while essentially devoid of any human myeloid cells. Similarly, the authors are also developing a myeloid only humanized mouse model that lacks T cells (Dr. Victor Garcia-Martinez, personal communication). These model systems could be invaluable in dissecting out the relative contribution of myeloid cells and $\mathrm{T}$ cells in the establishment and maintenance of HIV-CNS reservoirs in vivo.

Gene editing strategies have received special attention by the biomedical community in the last 2 years. In this respect, Dr. Kamel Khalili discussed the potential use of these strategies for eradicating HIV-1. These technologies include ZFN, TALENs, and the CRISPR/Cas9 system. These gene editing tools can target highly conserved regions of HIV-1 LTR or essential viral genes. The use of ZFNs and TALENs has proven to be costly and time-consuming. RNA-guided endonuclease Cas9 technology, called CRISPR/Cas9, has emerged as a simpler and more versatile technology to allow permanent removal of integrated HIV-1 proviral DNA in eukaryotic cells. Dr. Khalili has developed a strategy using CRISPR/Cas9 that effectively eliminates integrated copies of the viral DNA from several human cell culture models containing HIV-1 proviral DNA. This is a promising strategy which can be investigated toward eradication of HIV-1 from infected cells. Dr. Khalili, however, pointed out that there are some challenges with this approach including delivery, potential off-target cleavage, and cell-specific gene targeting, which need to be improved.

This issue also includes a meeting report authored by Dr. Bruce Brew and colleagues. The Division of AIDS Research at NIMH co-sponsored an affiliated event with the Burnet Institute and University of New South Wales at the 20th International AIDS conference in Melbourne, Australia, entitled "HIV Eradication-Will the Brain be Left Behind?" on July 18,2014 . The goal of the meeting was to bring together leading experts in NeuroAIDS and HIV-1 eradication to identify the challenges in the field and to develop partnerships to address those challenges. Several presentations discussed potential cellular sites of HIV-1 persistence in the brain such as microglial cells and astrocytes. The use of available animal models such the SIV macaque and myeloid only mouse model for studying CNS eradication was presented. Preliminary clinical studies on the impact of current reactivation strategies on neurocognitive outcomes were outlined. NIMH program outlined available funding opportunities through several NIMH and NIAID-sponsored FOAs to facilitate HIV-1 CNS reservoir research. The meeting ended with a discussion which identified barriers and risks associated with current approaches for targeting CNS reservoirs. Future needs for neurological studies and strategies to target CNS reservoirs were also discussed.

In summary, this special issue captures our current knowledge with regard to mechanisms of establishment of HIV-1 CNS reservoirs and challenges associated with targeting these reservoirs. We hope that this will serve as an important resource for the field and help stimulate further research in the area. We would also like to take this opportunity to thank all the authors for taking the time to contribute to this special issue. Finally, we would like to thank the Journal of NeuroVirology for devoting a special issue to this emerging research area. 